J. Sci. Univ. Kelaniya 10 (2015): 01-11

\title{
AN EXACT SOLUTION OF NON-NEWTONIAN PERISTALTIC FLOW IN A TUBE : RABINOWITSCH FLUID MODEL
}

\section{U.P. SINGH*}

\section{Department of Mathematics, Ansal Technical Campus, Lucknow, India}

\begin{abstract}
With the development of medical science, non-Newtonian fluids have taken on added significance with a variety of applications in real life. The flow of non-Newtonian fluids in tubes and pipes plays a vital role in daily life. The examples of such applications are medical instruments, human body, machines, etc. The study of such mechanisms with the Newtonian fluids has not been found satisfactory due to nonlinear behavior of stress strain relationship. In the present analysis, the study of peristaltic flow in a tube has been carried out taking into account the non-Newtonian fluid : Rabinowitsch fluid model. Considering the significant terms in Navier-Stokes equations, solutions have been derived for fluid flow in axial direction in terms of pressure gradient. Using the continuity of flow, and exact solution has been derived for fluid pressure at wall. To establish the applicability of the solution, results for pressure rise at wall, pressure gradient and streamlines have been presented graphically.
\end{abstract}

KEYWORDS: Non-Newtonian fluid, Peristaltic flow, Rabinowitsch fluid Model.

DOI: http://dx.doi.org/10.4038/josuk.v10i0.7991

\section{INTRODUCTION}

In last few decades, the analysis of the peristaltic flows has played very important role in the development of medical science, and therefore received much attention of the bio-mechanics researchers, though, it has various applications engineering and industries too. Some important daily-life applications of peristaltic flow in a tube are sanitary fluid transport and transport of corrosive fluids, blood flow in vessels, blood pumps in heartlung machines, etc., and some examples of peristaltic flow in medical science can be found

\footnotetext{
*Corresponding author Email: professorupsingh@gmail.com
} 
in gall bladder, gastro-intestinal tract, female fallopian tube and medicine injector equipment. A more detail can be also found in well recognized articles by Mekheimer (2005), Mernone and Mazumdar (2002), Srinivas and Gayathri (2009), Misra and Pandey (1995), Li and Brasseur (1993), Vajravelu, Sreenadh and Babu (2006).

It has been found that the use of high molecular weight polymer solutions (viscosity index improvers) can give rise to a non-Newtonian fluid with minimized sensitivity of change in shearing strain rate (Spikes, 1994). However, the use of additives changes the stress-strain relationship of the fluid. To study the flow properties of such fluids, many traditional non-Newtonian models such as couple stress, power law, micropolar and Casson models are employed. Amongst these models, Rabinowitsch fluid model (Singh, Gupta and Kapur, 2011) is an established model (Wada and Hayashi, 1971) to analyse the non-Newtonian behaviour of the fluid. The following stress-strain relation holds for Rabinowitsch fluid model for one dimensional fluid flow:

$$
\bar{\tau}_{r z}+\kappa \bar{\tau}_{r z}^{3}=\bar{\mu} \frac{\partial \bar{w}}{\partial \bar{r}}
$$

where $\bar{\mu}$ is the initial viscosity and $\kappa$ is the non-linear factor responsible for the nonNewtonian effects of the fluid which will be referred to as coefficient of pseudoplasticity in this paper. This model can be applied to Newtonian lubricants for $\kappa=0$, dilatant lubricants for $\kappa<0$ and pseudoplastic lubricants for $\kappa>0$. The advantage of this model lies in the fact that the theoretical analysis for this model is verified with the experimental justification by Wada and Hayashi (1971). After Wada and Hayashi, many researchers used this model to theoretically analyse the performance characteristics of bearing performance with non-Newtonian lubricants (Bourging and Gay, 1984; Hashmimoto and Wada, 1986; Lin, 2001). Recently, this model was used by Singh et al. (2011a,b; 2012a,b; 2013) to investigate the performance of different types of hydrostatic, hydrodynamic and squeeze film bearing systems. Therefore, by the two reasons - first, Rabinowitsch fluid model fits a wide range of viscosity data (Wada and Hayashi, 1971), and second, none of the investigators have studied the flow characteristics of peristaltic flow in a tube with the Rabinowitsch fluid model, the present investigation is motivated.

\section{ANALYSIS}

The Schematic diagram of a peristaltic flow through a uniform tube is shown in figure 1 . The fluid in the system is taken as incompressible non-Newtonian Rabinowitsch 
type (pseudoplastic or dilatant) fluid under isothermal condition. Consider the sinusoidal wave trains propagate with constant speed $c$ along the walls of the tube which let the fluid flow inside. The geometry of the wall surface $\left(\bar{r}_{w}\right)$ (cf. Figure 1) is defined as

$$
\bar{r}_{w}=\bar{r}_{i}+\bar{a} \sin \left(\frac{2 \pi}{\lambda}(\bar{z}-c \bar{t})\right)
$$

where, $\bar{r}_{i}$ is the tube radius at inlet, $\bar{a}$ is the wave amplitude, $\lambda$ is the wavelength, $c$ is propagation velocity and $\bar{t}$ is time.

The equations of motion of the flow in tube are

$$
\begin{aligned}
& \frac{1}{\bar{r}} \frac{\partial(\bar{r} \bar{u})}{\partial \bar{r}}+\frac{\partial \bar{w}}{\partial \bar{z}}=0 \\
& \rho\left(\frac{\partial \bar{u}}{\partial \bar{t}}+\bar{u} \frac{\partial \bar{u}}{\partial \bar{r}}+\bar{w} \frac{\partial \bar{u}}{\partial \bar{z}}\right)=-\frac{\partial \bar{p}}{\partial \bar{r}}-\frac{1}{\bar{r}} \frac{\partial\left(\bar{r} \bar{\tau}_{r r}\right)}{\partial \bar{r}}-\frac{\partial \bar{\tau}_{r z}}{\partial \bar{z}} \\
& \rho\left(\frac{\partial \bar{w}}{\partial \bar{t}}+\bar{u} \frac{\partial \bar{w}}{\partial \bar{r}}+\bar{w} \frac{\partial \bar{w}}{\partial \bar{z}}\right)=-\frac{\partial \bar{p}}{\partial \bar{z}}-\frac{1}{\bar{r}} \frac{\partial\left(\bar{r} \bar{\tau}_{r z}\right)}{\partial \bar{r}}-\frac{\partial \bar{\tau}_{z z}}{\partial \bar{z}}
\end{aligned}
$$

and the related boundary conditions are

$$
\begin{array}{lll}
\frac{\partial \bar{w}}{\partial \bar{r}}=0 & \text { at } & \bar{r}=0 \\
\bar{w}=0 & \text { at } & \bar{r}=\bar{r}_{w}
\end{array}
$$

where, $\bar{u}$ and $\bar{w}$ are velocity components in radial and axial directions, respectively; $\rho$ is the density of fluid, $\bar{\tau}_{a b}(a, b=r, z)$ are components of stress and $\bar{p}$ is pressure.

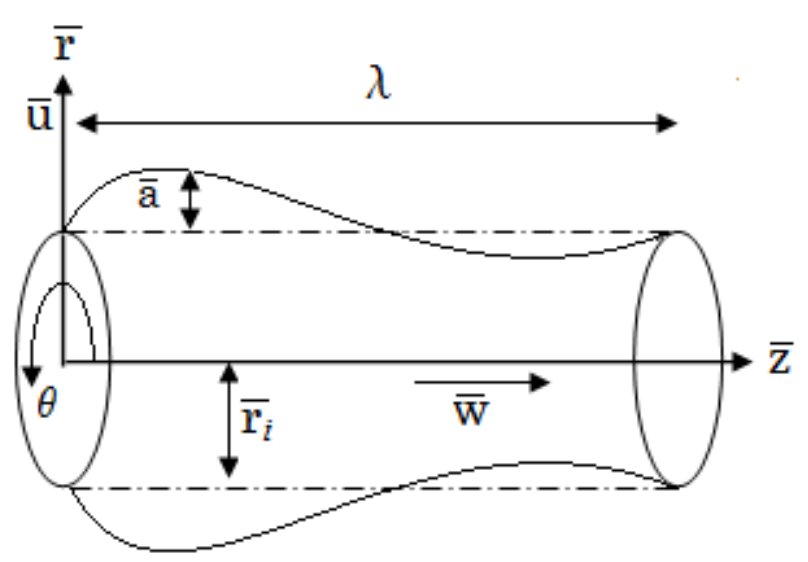

Schematic diagram of peristaltic flow in a circular tube. 
Taking the coordinate transformation $\tilde{z}=\bar{z}-c \bar{t}, \tilde{w}=\bar{w}-c$ together with the dimensionless parameters

$$
\begin{aligned}
& P=\frac{r_{i}^{2} \bar{P}}{c \lambda \mu}, \quad r=\frac{\bar{r}}{r_{i}}, \quad r_{w}=\frac{\bar{r}_{w}}{r_{i}}, R_{e}=\frac{\rho c r_{i}}{\mu}, t=\frac{c \bar{t}}{\lambda}, u=\frac{\lambda \bar{u}}{r_{i} c}, w=\frac{\tilde{w}}{c}, \\
& z=\frac{\tilde{z}}{\lambda}, \quad \alpha=\frac{c \mu}{r_{i}} \kappa, \quad \beta=\frac{k \lambda}{r_{i}}, \quad \delta=\frac{r_{i}}{\lambda}, \quad \phi=\frac{a}{r_{i}}, \quad \tau_{a b}=\frac{r_{i}}{c \mu} \tau_{a b}
\end{aligned}
$$

the equations ( $1-5)$ are transformed as

$$
\begin{aligned}
& \tau_{r z}+\alpha \tau_{r z}^{3}=\frac{\partial w}{\partial r} \\
& r_{w}=1+\phi \sin (2 \pi z) \\
& \frac{1}{r} \frac{\partial(r u)}{\partial r}+\frac{\partial w}{\partial z}=0 \\
& 0=-\frac{\partial p}{\partial r} \\
& 0=-\frac{\partial p}{\partial z}+\frac{1}{r} \frac{\partial\left(r \tau_{r z}\right)}{\partial r}
\end{aligned}
$$

where, the terms of order $\delta$ and higher have been dropped with the assumption that $\delta<1$, that is, the wavelength $(\lambda)$ is longer than the inlet radius $\left(r_{i}\right)$ of the tube, which is a common situation in the creeping flow in medical science such as endoscopy.

Further, the boundary conditions (6-7) are transformed to

$$
\begin{array}{lll}
\frac{\partial w}{\partial r}=0 & \text { at } & r=0 \\
w=-1 & \text { at } & r=r_{w}
\end{array}
$$

Integrating the equation (13) under the condition that the shear stress $\tau_{r z}$ vanishes along the axial line $r=0$, the following is obtained

$$
\tau_{r z}=\frac{1}{2} \frac{\partial P}{\partial z} r
$$


Solving equation (9) together with equation (16) and boundary condition, equations (14$15)$, expression for axial velocity $w$ is obtained as

$$
w=\frac{\partial p}{\partial z}\left(\frac{r^{2}-r_{w}{ }^{2}}{4}\right)+\alpha\left(\frac{\partial p}{\partial z}\right)^{3}\left(\frac{r^{4}-r_{w}{ }^{4}}{32}\right)-1
$$

The dimensionless instantaneous volumetric flow rate (cf. Appendix A) is obtained as

$$
Q=q+\frac{1}{4}\left(\phi^{2}+2\right)
$$

By substituting equation (17) in equation (18), equation of pressure gradient is obtained as

$$
\frac{d p}{d z}+\frac{1}{6} \alpha r_{w}^{2}\left(\frac{d p}{d z}\right)^{3}=-8\left(\frac{2 Q+r_{w}^{2}}{r_{w}^{4}}\right)
$$

In the limiting case, as $\alpha \rightarrow 0$, equation (19) reduces to the regular equation for Newtonian fluids

$$
\frac{d p}{d z}=-8\left(\frac{2 Q+r_{w}^{2}}{r_{w}^{4}}\right)
$$

The exact solution of equation (19) is obtained as using Tartaglia's method for cubic equations

$$
\frac{d p}{d z}=\left(-\frac{f_{1}}{2}+\sqrt{\left(\frac{f_{1}}{2}\right)^{2}+\left(\frac{f_{2}}{3}\right)^{3}}\right)^{1 / 3}+\left(-\frac{f_{1}}{2}-\sqrt{\left(\frac{f_{1}}{2}\right)^{2}+\left(\frac{f_{2}}{3}\right)^{3}}\right)^{1 / 3}
$$

where

$$
f_{1}=-\frac{48}{\alpha h^{4}}\left(1+\frac{2 Q}{h^{2}}\right), f_{2}=\frac{6}{\alpha h^{2}},
$$

The pressure rise can be calculated from equation (21) as follows

$$
\Delta p=\int_{0}^{1} \frac{\partial p}{\partial z} d z
$$

The streams lines can be obtained as

$$
u=\frac{1}{r} \frac{\partial \psi}{\partial z}, w=-\frac{1}{r} \frac{\partial \psi}{\partial r}
$$




\section{RESULTS AND DISCUSSION}

To analyse the effects of non-Newtonian fluids on the flow behaviour, the numerical results for pressure rise and flow rate have been calculated and plotted in the figures (1-3). The influence of non-Newtonian fluids is estimated with the different values of pseudoplastic parameter $\alpha(-0.1 \leq \alpha \leq 0.1)$.

Figure 1 shows the dimensionless amount of pressure rise at wall with respect to $\mathrm{Q}$ for different values of $\alpha$. It is observed that the pressure rise increase for increase in $\alpha$ and decrease with the decrease in $\alpha$. The pressure rise for pseudoplastic fluids, $\alpha>0$, is higher than the Newtonian fluids, whereas, for the dilatant fluids, $\alpha<0$, the pressure rise at wall is lower than that for a Newtonian fluid. The influence of non-Newtonian fluids increases rapidly with the increase in flow rate.

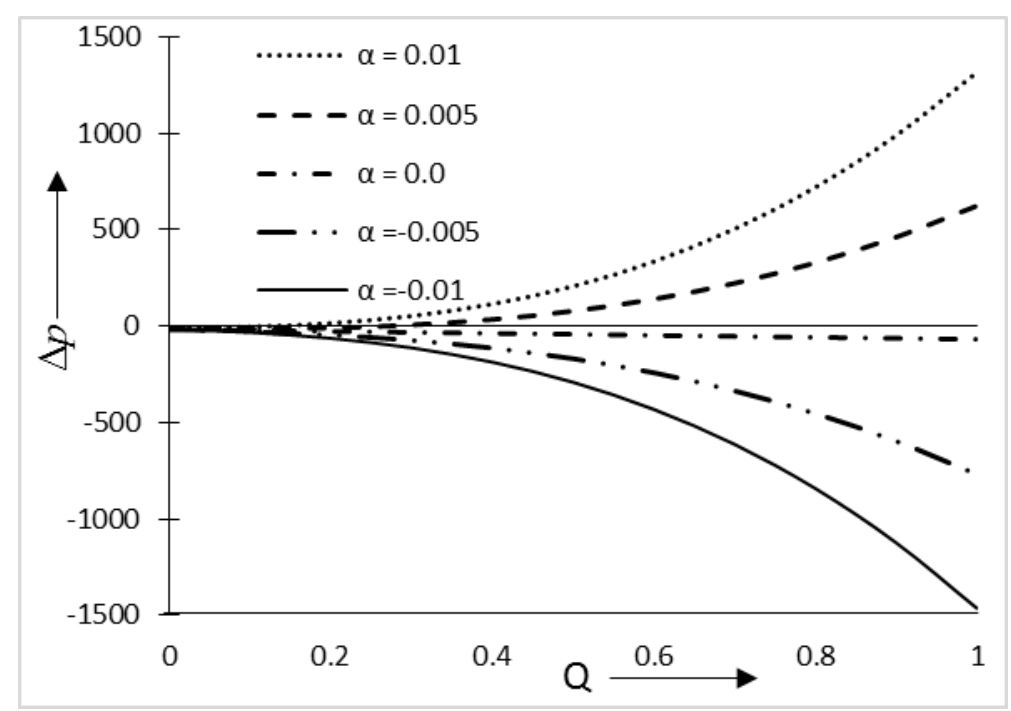

Figure 1: Variation of pressure rise at wall with respect to $\alpha$ for different values of flow rate. $\phi=0.5$.

Figure 2 shows the variation of pressure gradient along the axial direction. The effect of non-Newtonian fluids is analysed by means of taking different values of $\alpha$ into account. The pseudoplastic fluids $(\alpha>0)$ increases the pressure gradient while the case is reversed for dilatant fluids $(\alpha<0)$.

Figure 3 shows the fluctuation in velocity profile with respect to radial points for different values of $\alpha$. The effect of non-Newtonian fluids on velocity profile is demonstrated at a particular point $z=0.2$, which is just for the sake of the completeness 
and avoid the excess of plots. The similar variation holds for other values of $z$ the overall effect has been observed for pressure rise. Figures 3(a) -3(c) shows the stream lines of the flow.

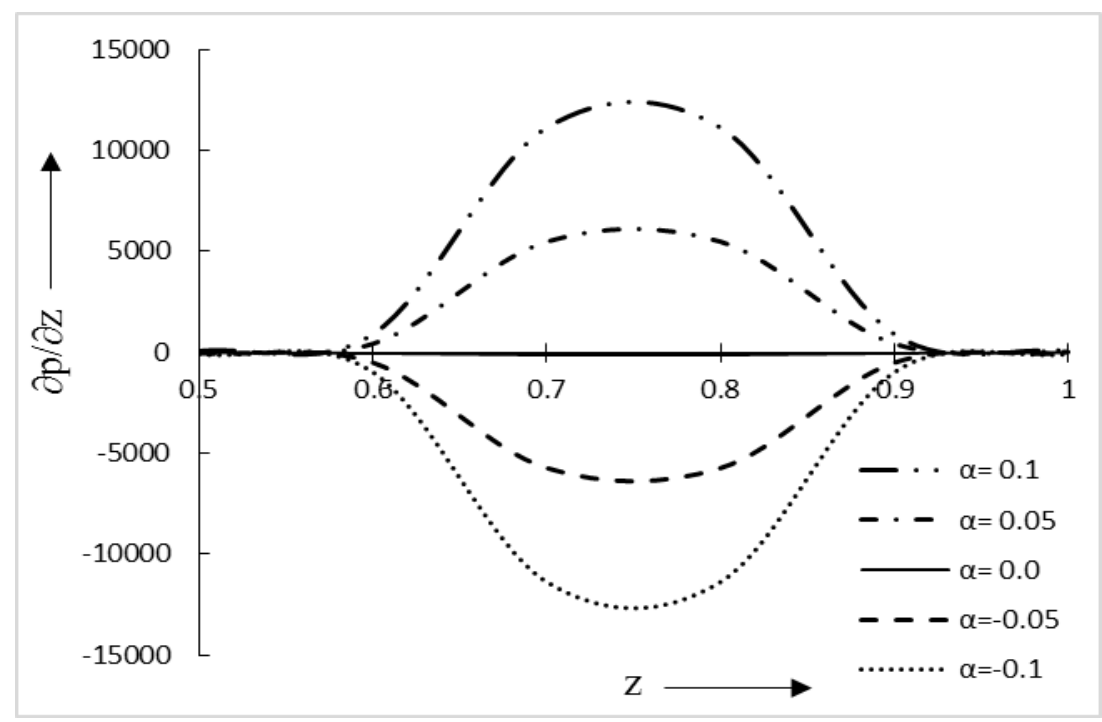

Figure 2: Variation of pressure gradient along axial direction

(z) for different values of $\alpha \cdot Q=0.5, \phi=0.5$.

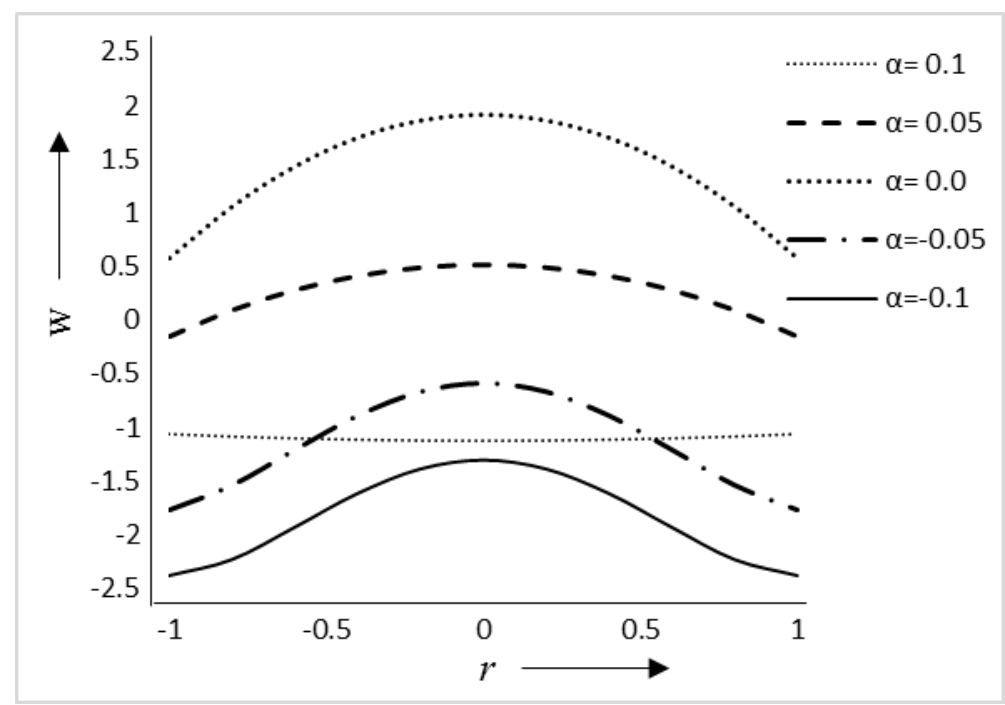

Figure 3: $\quad$ Variation of dimensionless velocity (w) for different values of $\alpha . Q=0.5, \phi=0.5$. 


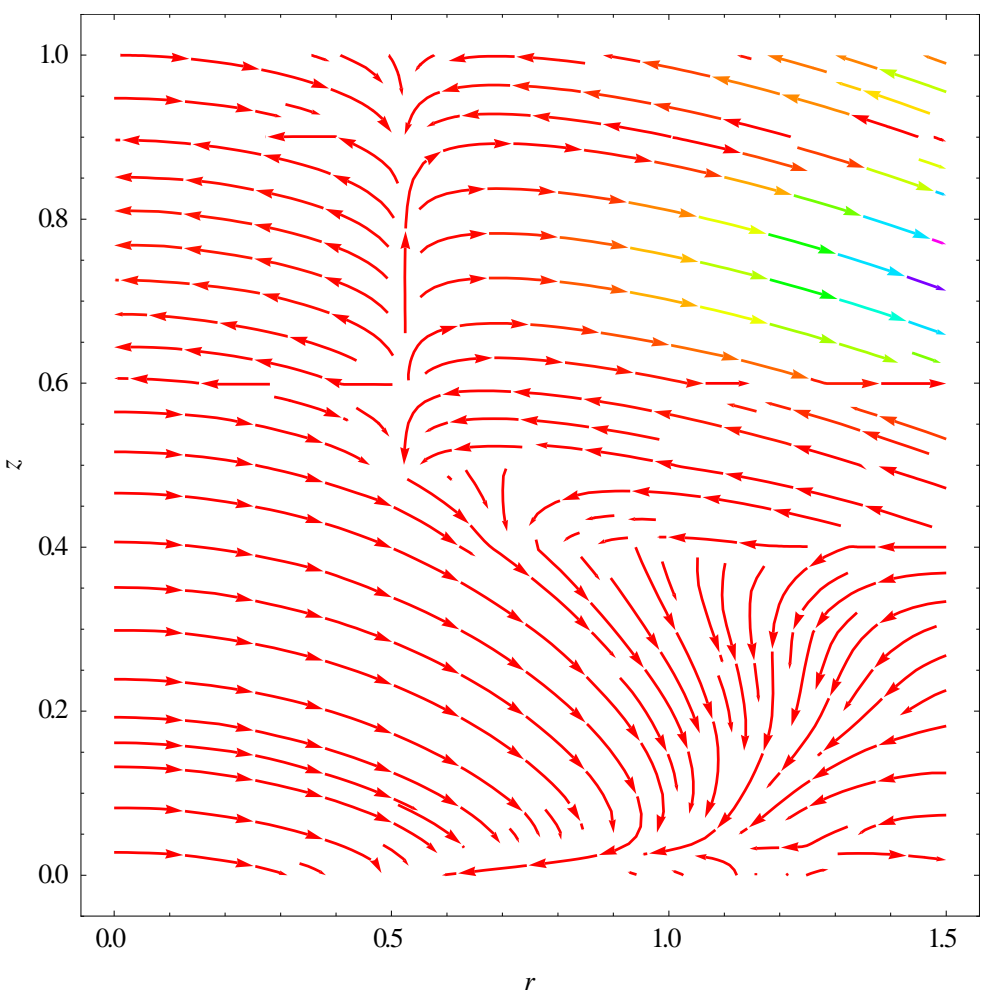

Figure 4 (a): Streamlines for $\alpha=-0.01$. $Q=0.5, \phi=0.5$.

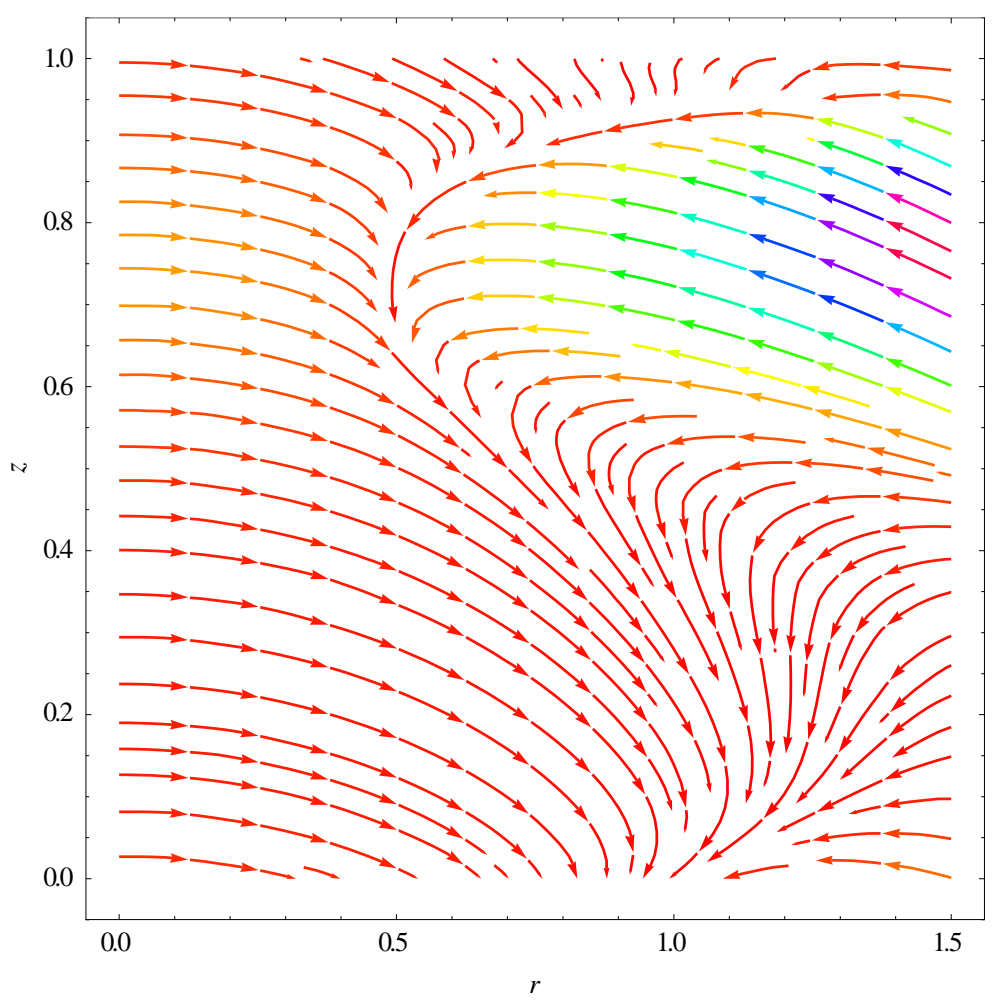

Figure 4(b): Streamlines for $\alpha=0 . Q=0.5, \phi=0.5$. 


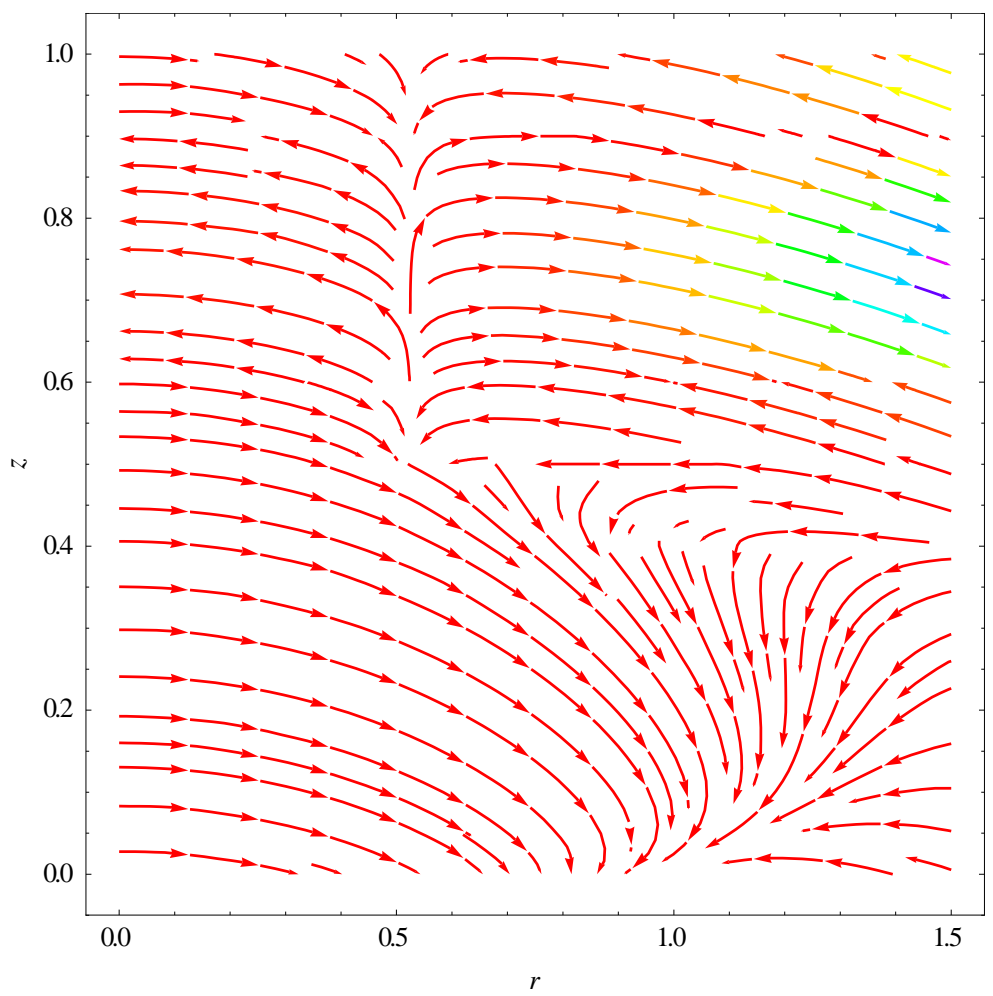

Figure 4(c): Streamlines for $\alpha=0.01 . Q=0.5, \phi=0.5$.

\section{CONCLUSION}

The influence of non-Newtonian pseudoplastic and dilatant type fluids on the nature of peristaltic flow in a tube has been analyzed by means of Rabinowitsch fluid model. Analytical expressions for radial velocity, pressure gradient and pressure rise at walls have been obtained. Both the pseudoplastic as well as dilatant fluids showed significant influence on the flow behavior, however the influence is observed to be dependent on instantaneous flow rate.

\section{REFERENCES}

Bourging P. and Gay B., 1984. Determination of the Load Capacity of Finite Width Journal Bearing by Finite Element Method in the Case of a Non-Newtonian Lubricant. ASME J. Tribol. 106, 285-290. http://dx.doi.org/10.1115/1.3260906

Hashmimoto H. and Wada S., 1986. The Effects of Fluid Inertia Forces in Parallel Circular Squeeze Film Bearing Lubricated with Pseudoplastic Fluids. ASME J. Tribol. 108, 282287. http://dx.doi.org/10.1115/1.3261177

Li M., Brasseur J.G., 1993. Non-steady peristaltic transport in a finite length tube. J. Fluid Mech. 248, 138-151. http://dx.doi.org/10.1017/S0022112093000710 
Lin J-R., 2001. Non-Newtonian Effects on the Dynamic Characteristics of one Dimensional Slider Bearings : Rabinowitsch Model. Tribology Letters. 10 (No. 4), 237-243. http://dx.doi.org/10.1023/A:1016678208150

Mekheimer K.S., 2005. Peristaltic transport of a non-Newtonian fluid through a uniform and nonuniform annulus. The Arabian J. Sci. Engg. 30 (1A), 70-83.

Mernone A.V. and Mazumdar J.N., 2002. A Mathematical Study of Peristaltic Transport of a Casson Fluid. Math. and Comp. Modelling. 35, 895-912. http://dx.doi.org/10.1016/S0895-7177(02)00058-4

Misra J.C. and Pandey S.K., 1995. Peristaltic Transport in a Tapered Tube. Mathl. Comput. Modelling. 22(8), 137-151. http://dx.doi.org/10.1016/0895-7177(95)00162-U

Singh U.P., 2013. Application of Rabinowitsch Fluid Model to Pivoted Curved Slider Bearings. Archive of Mechanical Engineering. LX(2013), 247 - 267.

Singh U.P., Gupta R.S. and Kapur V.K., 2011 a. On the steady performance of hydrostatic thrust bearing: Rabinowitsch fluid model. Tribol. Trans. 54(5), 723-29.

http://dx.doi.org/10.1080/10402004.2011.59754

Singh U.P., Gupta R.S. and Kapur V.K., 2011 b. Effects of Inertia in the Steady State Pressurised Flow of a non-Newtonian Fluid Between two Curvilinear Surfaces of Revolution: Rabinowitsch fluid model. Chemical and Process Engineering. 32 (4), 333-349. http://dx.doi.org/10.2478/v10176-011-0027-1

Singh U.P., Gupta R.S. and Kapur V.K., 2012 a. On the Steady Performance of Annular Hydrostatic Thrust Bearing: Rabinowitsch fluid model. ASME Journal of Tribology. 134 (4), 1-5. http://dx.doi.org/10.1115/1.4007350

Singh U.P., Gupta R.S. and Kapur V.K., 2012 b. On the Squeeze Film Characteristics between a Long Cylinder and a Flat Plate: Rabinowitsch fluid model. IMechE Journal of Engineering Tribology. 227 (1), 34-42. http://dx.doi.org/10.1177/1350650112458742

Spikes H.A., 1994. The behaviour of lubricants in contacts: current understanding and future possibilities. Proc. IMechE. Part-J : J. Eng. Tribol. 208(1), 3-15.

Srinivas S., Gayathri R., 2009. Peristaltic transport of a Newtonian $\$$ fluid in a vertical asymmetric channel with heat transfer and porous medium. Applied Mathematics and Computation. 215, 185-196. http://dx.doi.org/10.1016/j.amc.2009.04.067

Vajravelu K., Sreenadh S. and Babu V.R, 2006. Peristaltic transport of a Herschel-Bulkley fluid in contact with a Newtonian fluid. Quart. Appl. Math. 64, 593-604.

http://dx.doi.org/10.1090/S0033-569X-06-01020-9

Wada S. and Hayashi H., 1971. Hydrodynamic lubrication of journal bearings by pseudoplastic lubricants (part II, experimental studies). Bulletin of JSME. 14 (No. 69), 279-86. http://dx.doi.org/10.1299/jsme1958.14.279 


\section{APPENDIX}

The instantaneous volumetric flow rate is

$$
\tilde{Q}=2 \pi \int_{0}^{\bar{r}_{w}} \bar{r} \bar{w} d \bar{r}
$$

Using the time relaxed system $\tilde{w}=\bar{w}-c, \quad \tilde{z}=\bar{z}-c \bar{t}$ and taking the time-mean flow $\bar{Q}=\frac{1}{T} \int_{0}^{T} \tilde{Q} d \bar{t}$ over the time $T=\lambda / c$, equation (18) can be written as

$$
\bar{Q}=\bar{q}+\pi c\left(\bar{r}_{i}^{2}+\frac{1}{2} \bar{a}^{2}\right)
$$

which can be written in dimensionless form as

$$
Q=q+\frac{1}{4}\left(\phi^{2}+2\right)
$$

where, $q=\frac{\bar{q}}{2 \pi c r_{i}^{2}}, Q=\frac{\bar{Q}}{2 \pi c r_{i}^{2}}$ 